\title{
À l'école de la République française : regard critique sur une institution
}

Thierry Pardo

\section{(2) OpenEdition \\ Journals \\ Édition électronique \\ URL : http://journals.openedition.org/ere/3281 \\ DOI : $10.4000 /$ ere.3281 \\ ISSN : 2561-2271 \\ Éditeur}

Centr'ERE

Référence électronique

Thierry Pardo, "À l'école de la République française : regard critique sur une institution », Éducation relative à l'environnement [En ligne], Volume 7 | 2008, mis en ligne le 20 septembre 2008, consulté le 21 février 2020. URL : http://journals.openedition.org/ere/3281 ; DOI : 10.4000/ere.3281 


\title{
À l'école de la République française : regard critique sur une institution
}

\author{
Thierry Pardo
}

1 L'éducation relative à l'environnement est-elle compatible avec les valeurs issues des fondements historiques de l'Éducation nationale en France? La formation des enseignants ne souffre-t-elle pas d'une volonté d'uniformisation peu favorable à la diversité des situations offertes par l'éducation relative à l'environnement?

2 Tout au long de cet article, j'essaierai de faire ressortir l'étroite conception de l'éducation, limitée à l'horizon des murs de la classe, que porte l'institution scolaire. Je mettrai de l'avant une vision de l'environnement bio-régionaliste, proche de ses racines locales, également négligée tant dans le quotidien des élèves que dans la formation des enseignants. Ces deux points sont les fondements du regard critique que je porte sur l'institution éducative et qui à mon avis devraient davantage interpeller ses acteurs.

\section{Les portes d'une école « hors sol »?}

3 J'avais douze ans. La ville de Marseille venait d'être bloquée par une tempête de neige pour la première fois depuis vingt ans. Les trente centimètres avaient suffi à paralyser la ville et le premier chasse-neige se trouvait dans les Alpes. Rien à faire. Il fallait attendre que ça fonde. Les transports en commun étaient bloqués, mais heureusement mon école n'était qu'à trente minutes de marche. Il y avait donc pas mal d'élèves absents ce jour-là. Tous ceux qui ne pouvaient pas se rendre à l'école à pied étaient restés chez eux. À neuf heures, nous avons traversé la cour pour nous rendre au laboratoire de «sciences naturelles". Nous nous poussions dans la neige pour nous faire tomber. Certains faisaient des boules pour les jeter sur les autres.

Débarrassés de nos manteaux, nous nous installâmes dans l'excitation générale, mais nous n'avions d'yeux que pour ce blanc matelas de neige qui nous invitait à toucher, rouler, explorer. Dans ma classe, certains élèves arrivaient directement d'Algérie ou de Tunisie. Pour la majorité, ces familles immigrées n'avaient sans doute pas les moyens de partir découvrir les sports d'hiver. Malgré un équipement largement inadapté, ces 
condisciples des pays chauds semblaient être les plus friands de cette nouvelle occasion de jeu et de découverte. La classe commença. Dans la suite logique du programme entamé la semaine d'avant, l'enseignante nous fit un brillant exposé sur le calcaire et la géologie en général.

5 En repensant à cette expérience, il me semble que c'était là une occasion ratée d'éducation relative à l'environnement. Encore avions-nous eu la chance d'avoir traversé la cour pour nous rendre au laboratoire! D'autres avaient suivi les couloirs pour se retrouver en salle d'étude. Là, ils avaient dû patienter une heure à cause de l'absence d'un professeur.

6 Mais au-delà de l'anecdote, je m'interroge sur les causes de cette expérience. Est-il possible que le système scolaire en France (comme ailleurs ?) évolue dans une sorte de logique « hors sol »? L'environnement naturel, urbain et social a-t-il une influence sur les programmes et le contenu des cours? Ce genre d'expérience de mon enfance au lendemain d'une tempête de neige n'est-il pas dû à une formation inadéquate des enseignants, déconnectée de son environnement? Si l'on s'intéresse d'un peu plus près à cette question de la formation des enseignants, sans a priori et sans tenter de débusquer le diable dans les détails, on peut être légitimement surpris. À la question : «Est-il normal que l'on puisse être un professeur d'anglais diplômé, titulaire et permanent, sans jamais avoir voyagé dans un pays anglophone? », le système français répond « oui ». Chacun appréciera. Or, comment une formation « hors sol " pourraitelle générer un enseignement soucieux et imprégné de son environnement? Si un professeur d'anglais n'a jamais été en contact direct avec un milieu anglophone, que peut-il transmettre de cette mosaïque de cultures? Et qu'en est-il pour les autres professeurs?

7 En outre, existe-t-il une culture d'éducation relative à l'environnement suffisamment établie pour que les différentes pratiques puissent être repérées comme telles par les enseignants ? Le ministère de l'Éducation nationale possède-t-il une connaissance assez profonde au sujet de l'éducation relative à l'environnement pour pouvoir la reconnaître dans les projets existants et en favoriser le développement ?

Les formateurs dans ce système sont également spécialisés, parfois si spécialisés qu'ils ne disposent d'aucune culture générale, encore moins d'une culture locale endogène considérée comme 0070erturbatrice de l'idéologie pédagogique réductrice dans laquelle ils ont été formés, coupant l'école de la vie. (Wicht et Mondjanagni, 2004)

8 Autant de questions qui prennent tout leur sens en cette période, puisque « l'éducation à l'environnement pour un développement durable » est entrée récemment dans les cursus des écoles, collèges et lycées français (Ministère de l'Éducation nationale, de l'Enseignement supérieur et de la Recherche, 2004).

9 La question se pose avec plus d'acuité encore si l'on considère que l'école se doit de favoriser la rencontre entre un élève et un enseignant, entre deux existences, avec tout ce que cela implique de différences culturelles, de différences de centres d'intérêts, de préoccupations, de rythmes d'apprentissages ou de projets de vie, en somme de deux êtres imprégnés de leur rapport au monde qui, le temps d'une année, se rencontrent pour s'enrichir mutuellement. Cette rencontre pose la question de la légitimité d'enseigner. 


\section{La légitimité d'enseigner}

10 La question de la légitimité d'une personne autorisée à transmettre le savoir se pose depuis longtemps. Dans toutes les sociétés vernaculaires (comme les nomme Majid Rahnema, 2004 - mais on pourrait préférer le terme d'« écosystème culturel » comme le mentionne le Rapport de la Commission mondiale de la culture et du développement, Notre diversité créatrice, Pérez de Cuéllar et coll., 1996), l'autorité des « anciens » pour transmettre un savoir maîtrisé s'appuie sur leurs expériences et leurs réflexions. Cette autorité est souvent reconnue de la plupart parce que ces «anciens» partagent quotidiennement la vie du village. Ainsi, ils ont l'occasion de démontrer sagesse et maîtrise plusieurs fois par jour, ce qui leur confère une légitimité pour transmettre ces qualités reconnues par tous comme utiles. Quand un enfant désire apprendre à faire un panier, ses parents peuvent lui conseiller d'aller voir tel ou tel artisan reconnu pour son art et, pourquoi pas, son habileté pédagogique. De plus, l'utilité d'un panier dans la vie quotidienne ne fait aucun doute.

11 Dans notre Éducation nationale, les choses sont différentes. L'enfant ne choisit pas le fait d'aller à l'école puisque l'État a rendu la scolarité quasi universelle. Il ne choisit pas non plus les adultes chargés de l'enseignement, pas plus que les sujets d'étude, au moins dans les premiers temps de sa vie d'écolier. Le contexte pédagogique scolaire sélectionne de fait les sujets et les pédagogies qui les portent.

Devant le spectacle affligeant, devant le désarroi du monde, beaucoup d'esprits mûrs se demandent si nous n'avons pas fait fausse route en condamnant le cerveau de nos enfants et de nos jeunes à un régime exclusif de papier noirci [...]. (MarieVictorin, 1935, p. 11)

Ainsi, un scénario catastrophe autorise à penser qu'un enfant n'aimant pas être assis en classe puisse étudier un sujet dont il ne sent pas l'utilité avec un professeur qu'il n'apprécie pas. "J'ai toujours pensé que l'école, c'était d'abord les professeurs. Qui donc m'a sauvé de l'école, sinon trois ou quatre professeurs? ? (Pennac, 2007, p. 57).

La légitimité de l'enseignant ne doit des comptes qu'à l'institution ou au ministère. Seul le diplôme suffit pour être titularisé tout au long de son parcours professionnel. Que penser alors de l'autodidaxie, des parcours de vie, des aventures ou expériences, des talents individuels et de la place qui leur est dévolue dans l'Éducation nationale ? On leur préférera sans doute le parcours des personnes qui ont suivi le cursus à la lettre sans plus d'égarements. Cela ne veut pas dire qu'il faille condamner les professeurs qui ont suivi ce genre de parcours, mais l'on pourrait légitimement s'interroger sur l'esprit d'ouverture extra-scolaire qui en résulte et sur l'intérêt qu'un professeur porte à son environnement. Dès le départ, le système montre qu'il est inutile pour un individu de faire preuve de connaissances à propos des différentes dimensions de la vie. L'aspect technique de la connaissance suffit à vous asseoir dans un fauteuil d'enseignant. L'éducation se résume alors à un transfert de connaissances isolées bien loin des préoccupations interdisciplinaires et multidimensionnelles de l'éducation relative à l'environnement.

14 Dans ces conditions, l'élève ne pourra faire valoir ses choix qu'à l'intérieur des aspects techniques de la connaissance. Quand un élève rencontre un conseiller d'orientation (diplômé comme il se doit de l'Éducation nationale), quelles sont les perspectives qui lui sont offertes? Le travail dans la solidarité internationale, l'éducation relative à l'environnement, l'agriculture biologique, le grand reportage, la presse indépendante, 
les carrières artistiques, le métier d'ingénieur du son, le spectacle de rue, etc.? Non, tous ces sujets existant en dehors des cursus scolaires habituels sont souvent peu connus et peu valorisés. A-t-on seulement pensé combien nos vies seraient ternes sans les artistes, musiciens, photographes du bout du monde, marginaux, voyageurs, sportifs, aventuriers de tous poils? Pourtant quand un jeune entame une démarche qui le conduira vers les choix fondamentaux de sa vie, on lui parlera plus volontiers de baccalauréat, de BTS (brevet de technicien supérieur), de CAP (certificat d'aptitude professionnelle) ou d'université et on l'encouragera surtout à renoncer à la moindre prise de risque de nature à ralentir sa trajectoire «normale » de réussite scolaire. Pour prendre le seul sujet de l'éducation relative à l'environnement, la France (contrairement à beaucoup de pays comme le Québec, les États-Unis, la Bolivie, le Brésil, Israël, etc.) n'en offre qu'une présence marginale dans les programmes universitaires. Il est donc très difficile d'établir une équivalence formelle avec les diplômes étrangers. Le métier d'« éducateur à l'environnement » ne figure pas sur la liste des métiers possibles à l'Agence nationale pour l'emploi (ANPE). Les conseillers en orientation sont-ils capables de parler de ce genre de professions? Vont-ils orienter les élèves vers des universités étrangères ou une organisation non gouvernementale?

Comment s'étonner alors de la place qu'occupent les préoccupations environnementales dans notre pays?

\section{Visite à l'Institut universitaire de formation des maîtres (IUFM) de Rodez}

Pour vérifier le caractère que je supposais « hors sol » de la formation des enseignants, je décidai d'aller rencontrer professeurs et étudiants de l'Institut universitaire de formation des maîtres (IUFM) de Rodez (Aveyron). Bien sûr, cette visite n'a pas la légitimité d'une enquête approfondie, il s'agit d'une démarche de recherche exploratoire. Mais les résultats obtenus laissent entrevoir que l'hypothèse de départ pourrait être confirmée par une étude plus poussée.

Cette investigation m'a permis de rencontrer en entrevues individuelles trente-huit personnes appartenant à trois groupes différents : dix-huit élèves, futurs professeurs des écoles ; dix-huit enseignants déjà en exercice depuis plusieurs années effectuant un stage dans le cadre de la formation continue et enfin, deux formateurs de l'IUFM. Voici les quatre questions qui leur ont été posées :

- Y a-t-il une partie de votre formation consacrée à la connaissance de l'environnement régional de l'IUFM?

- Pensez-vous que si votre IUFM était dans un autre environnement naturel ou urbain, l'enseignement y serait différent?

- Pour vous, qu'est-ce que l'éducation à l'environnement?

- Pouvez-vous me citer un(e) chercheur(e), pédagogue ou auteur(e) contemporain(e) qui ait parlé d'éducation à l'environnement?

18 Les réponses à mes questions ont été très différentes suivant le groupe.

19 À la première question, les formateurs eurent une réponse enthousiaste : La formation est largement consacrée à la connaissance de l'environnement de l'IUFM puisque dès le premier jour un rallye découverte est organisé dans la ville de Rodez. Plusieurs partenaires, tels les archives départementales, la société des lettres, les compagnons 
tailleurs de pierre qui restaurent la cathédrale [...] interviennent dans ce rallye. Puis, au cours de la formation, des projets individuels en relation avec l'environnement et le patrimoine sont demandés [...].

Bien sûr, la deuxième question s'inscrivait dans cette même logique et j'étais assuré que la charte du patrimoine et les différents intervenants, mentionnés par l'un des répondants, garantissaient l'ancrage environnemental de l'IUFM. Un formateur avait même coordonné l'année précédente un ouvrage, L'Aveyron une histoire, pour permettre aux élèves-maîtres d'avoir des références locales dans leur rôle d'enseignant. Une formatrice souligna le fait que les problèmes sociaux de certains IUFM se situant dans les environnements urbains « difficiles » ou dans les campagnes françaises en voie de désertification forcent les portes des écoles et qu'il était impossible de ne pas en tenir compte dans le cadre de la formation.

La troisième réponse de ces formateurs s'avéra encore la plus précise et la plus décidée : Éduquer à l'environnement, c'est appréhender le contexte dans lequel évolue l'humain pour y vivre en harmonie. Pour avoir rencontré ces personnes, je pense qu'on peut lire dans cette réponse l'attachement de ces formateurs au territoire aveyronnais et à ses dimensions culturelles ou revendicatives telle la défense de l'occitan dans une conception bio-régionaliste.

22 La quatrième question posa un problème à tous les groupes interrogés. Mais là encore la réponse la plus fournie émergea des formateurs: Albert Jacquard, Claude Allègre, ancien ministre de l'Éducation nationale [...].

23 Le deuxième groupe de personnes interrogées, les professeurs diplômés en formation continue, ne semblait pas attendre grand-chose tant de la formation initiale que de leur stage actuel et somme toute, de la formation en général. Ils me répétèrent plusieurs fois que l'enseignant était très largement livré à lui-même et que la formation n'apportait qu'une part insignifiante dans l'expérience des enseignants. Le fait que la formation laisse une large part à la démarche individuelle de ses étudiants pourrait être une bonne nouvelle. Cela favoriserait les initiatives et les projets personnels. Mais n'imposant aucune obligation reliée à l'inventivité pédagogique, il semble que le système laisse trop souvent place à l'absence d'initiative.

La première question ( $\mathrm{Y}$ a-t-il une partie de votre formation consacrée à la connaissance de l'environnement de l'IUFM ? ») fit l'objet d'une majorité écrasante de non représentant $90 \%$. Le rallye découverte ne semblait pas avoir marqué les esprits. Une enseignante se souvint d'une course d'orientation durant sa formation.

La deuxième question reçut, elle aussi, une quasi-totalité de non allant dans la logique de la première réponse. En revanche, ces enseignants pratiquants semblaient être plus à l'aise pour donner une définition de l'éducation à l'environnement. Ils s'appuyaient sur des exemples d'initiatives mises en place dans les classes: activités physiques de pleine nature, observation, citoyenneté, respect et protection de la nature, sensibilisation, recyclage des déchets [...]. Ces réponses ne paraissent pas s'appuyer sur une connaissance consciente et théorisée donnant accès à une vision globale. Elles relèvent plus spontanément d'une expérience de terrain.

Enfin, la quatrième question reçut elle aussi une immense majorité de non. Seuls Hubert Reeves et Nicolas Vanier vinrent à l'esprit de nos enseignants.

Les élèves futurs professeurs arrivent quant à eux, en queue des réponses. Ils donnèrent de façon quasi unanime une réponse négative à toutes les questions. Là, le 
rallye de la première journée fut évoqué. Mais il était facultatif et surtout réservé aux élèves arrivant d'autres régions. Ainsi la plupart n'y avaient pas participé. Pour la définition de l'éducation à l'environnement, les mêmes mots clés, intuitivement retrouvés apparurent : protection, respect, sensibilisation, les gestes quotidiens [...]. Je n'ai eu aucune réponse à la question des pédagogues connus.

Ainsi, sur trente-huit personnes, seuls deux formateurs font explicitement référence à l'influence de l'environnement sur la formation. Malheureusement, cette vision ne semble pas être partagée par l'ensemble des élèves et stagiaires interrogés. Pour le moins, on peut penser que même si une dimension environnementale existe au sein de l'IUFM de Rodez, la grande majorité des apprenants n'en est pas consciente. Les réponses à la question relative aux auteurs et pédagogues montrent que les pratiques se mettent en place essentiellement de façon intuitive. Bien sûr, dans ce genre d'institut on peut légitimement s'attendre à ce que tout le monde ait une vision de l'éducation et une conception, aussi floue soit-elle, de l'environnement. Mais nous sommes sans doute loin d'une culture partagée de l'éducation relative à l'environnement qui soit significative.

\section{Unité et uniformité}

29 La construction de la France s'est réalisée dans la douleur historique. Il suffit pour cela de rappeler que les frontières actuelles ne datent que de 1945. Les revendications régionales corses, occitanes, basques, bretonnes, alsaciennes, mais encore bien plus mahoraises, kanaks ou guyanaises ont poussé les "pères de la République » à donner une culture commune à tous les Français.

Le royaume de France (regnum Francae) n'existe dans les textes qu'à partir du XIIIème siècle, mais le déroulement finaliste de l'histoire d'une France toujours déjà là, occulte les identités historiques hétérogènes des États antérieurement à leurs annexions, et donc le caractère multiculturel et multilingue du royaume. (Citron, 2004, non paginé)

C'est dans cette logique que les langues régionales ont été prohibées durant plusieurs années dans les établissements scolaires, mais aussi que l'affectation des professeurs des collèges et lycées est nationale, ne tenant pas compte de la région de provenance de ces derniers. Les enseignants du premier degré en revanche profitent d'une affectation régionale, ce qui favorise sans doute les approches de terrain et les expériences concrètes que le sondage à l'IUFM met en lumière. Mais cette volonté d'uniformisation a tout de même fortement imprégné les programmes d'histoire. Ainsi, un élève de Marseille reçoit la même histoire de France qu'un élève de Lille. Seule l'idéologie peut réussir ce genre de contorsions improbables. La cité phocéenne, province de Rome et son port ouvert sur la Méditerranée serait sans doute plus proche de l'histoire de Gênes que de celle des Flandres, de leurs tapisseries et de leurs bassins industriels et miniers.

Les historiens républicains, qui voyaient la France comme la lumière du monde, comme une patrie-Messie ont élaboré un schéma du passé destiné à nationaliser les Français et à forger leur patriotisme. Le récit historique installait l'imaginaire d'une France homogène, une, indivisible, essence métahistorique mystérieusement présente dans une Gaule mythique originelle. (Citron, 2004, non paginé)

Pour asseoir donc les fondements de l'État, il fallait donner par l'éducation un sentiment national infaillible qui devait convaincre tout le monde, y compris les habitants des régions colonisées. L'histoire de l'enseignement dans l'Afrique 
francophone offre un bel exemple de cette volonté homogénéisatrice. L'éducation devait se décontextualiser de son enracinement local, linguistique, historique, naturel. En somme l'éducation devait, et nous en voyons encore les traces aujourd'hui, se dérouler de façon invariable et donc se détacher de son environnement immédiat. On pourrait constater à cet effet que cela correspond au processus classique de construction d'une nation.

[...] la nation est une construction du haut vers le bas, une construction qui part des élites de l'État pour s'appliquer aux populations d'un territoire clairement délimité, celui de l'État-nation. À cet égard, le projet moderne envisage la nation notamment comme un effort constant pour s'arracher aux rites obscurs, aux croyances ancestrales, ainsi qu'aux hiérarchies locales et arbitraires, et maintenir un idéal de progrès. (Wicht et Mondjanagni, 2004)

Mais même dans ce contexte général, la France fait figure d'exception en Europe. Sur le seul plan de l'officialisation des langues locales, on pourrait rappeler ici que la Norvège, l'Irlande, la Belgique, l'Espagne, la Suisse, par exemple, possèdent toutes plusieurs langues officielles bien que la population de ces pays y soit moins nombreuse qu'en France. L'Éducation nationale ne semble donc pas trouver son origine et sa légitimité dans un effet de zoom vers les connaissances locales, mais plus dans le grand-angle de l'unité nationale.

Cela ne devrait sans doute pas empêcher les enseignants d'aborder les macroproblèmes environnementaux comme les changements climatiques ou la destruction de la couche d'ozone. En effet, selon les témoignages recueillis à l'IUFM, cette dimension semble être la plus compatible avec les volontés ministérielles, car le plus souvent, proche des consciences citoyennes et des petits gestes qui y sont associés. L'éducation relative à l'environnement dans sa version locale, bio-régionaliste, se présente encore trop souvent comme une entrave au sentiment national. Le pas de « citoyen français » à "citoyen du monde" paraît s'accorder avec beaucoup plus de facilité à la culture étatique de «l'école de la République ». La visite à l'IUFM a confirmé que le diplôme d'enseignant, étendard de l'unité républicaine ne pouvait tenir compte du milieu local puisqu'il est supposé être valable partout en France, outre-mer compris. Les institutions éducatives semblent être plus enclines à se laisser émouvoir par les macroproblèmes qui ramènent à une conscience et à une responsabilité globales du citoyen, pas très éloignées du sentiment civique cher à la République. Ce qui n'empêche pas certains professeurs de revendiquer des appartenances locales et d'orienter leurs cours dans ce sens. Cela se voit notamment chez les enseignants du primaire qui ont la possibilité d'exercer dès le départ dans leur région de résidence. Mais il n'en reste pas moins vrai que l'épouvantail du communautarisme est certainement celui qui est le plus souvent brandi par les représentants des institutions pour légitimer un cursus national décontextualisé.

En tant que cadre politique, social et culturel unitaire et homogène se construisant $\mathrm{du}$ haut vers le bas et en coïncidence parfaite avec l'État, il est clair que la nation est un modèle peu adapté à l'idée de diversité culturelle (qui est ainsi plutôt ressentie en tant que retour en arrière à une situation prémoderne). (Wicht et Mondjanagni, 2004) 


\section{L'invisible éducation relative à l'environnement}

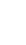

'enironnement semble être bien tortueux. Les nombreux pièges seront sans doute difficiles à éviter. Notre culture de système scolaire unifié, uniformisé, conçu pour porter haut les valeurs de la République, aura certainement beaucoup de mal à ouvrir ses portes pour laisser les enfants jouer dans la neige. On pourra remarquer que si plusieurs pays nomment le cadre institutionnel de leur système éducatif «ministère de l'Éducation ", la France choisit plutôt « ministère de l'Éducation nationale ». Encore un hasard? Les priorités d'alphabétisation, d'intégration des cultures dans la laïcité, la peur des communautarismes et du morcellement sont autant de freins endémiques à une réelle implantation de l'éducation relative à l'environnement dans les programmes scolaires français. Il serait sans doute intéressant d'étudier le parallèle qui semble exister entre l'ostensible silence sur l'apport des immigrations de tout temps dans l'identité nationale, et le silence sur l'apport de ces « étrangers de l'intérieur » que sont les cultures locales pour la construction de cette même identité. Ces deux silences se conjuguent pour rendre plus difficile encore l'accès à une vision de l'éducation ouverte, diverse, multiculturelle et écologique à l'intérieur de l'institution éducative. Aujourd'hui, le débat semble se cristalliser autour des célèbres fondamentaux «lire, 
écrire, compter... » dans une logique d'intégration au marché du travail. L'éducation relative à l'environnement avec ses diverses dimensions - naturaliste, critique, ethnographique, etc. - pourrait bien être très éloignée des urgences liées aux ratés intégrationnistes de la République.

Et si les fameux «fondamentaux » n'étaient pas « lire, écrire, compter... », mais vivre ensemble avec détermination, courage, ouverture d'esprit dans son environnement avec un sentiment de respect et de responsabilité ? Le repli sur lui-même d'un système auto-référencé ne pourrait-il pas nous enfermer dans une espèce d'« éducation hors sol », inapte à faire émerger les qualités ou les rêves nécessaires aux fabuleux destins de ses futurs adultes?

\section{BIBLIOGRAPHIE}

Citron, S. (2004). Dénationaliser l'histoire de France. Libération, le jeudi 30 décembre 2004.

Marie-Victorin, Frère. (1935). Flore laurentienne. Montréal : Imprimerie de La Salle.

Ministère de l'Éducation nationale, de l'Enseignement supérieur et de la Recherche. (2004). Bulletin officiel no. 28 du 15 juillet 2004 : Généralisation d'une éducation à l'environnement pour un développement durable (EEDD) - Rentrée 2004. Paris : Ministère de l'Éducation nationale, de l'Enseignement supérieur et de la Recherche.

Pennac, D. (2007). Chagrin d'école. Paris : Gallimard.

Pérez de Cuéllar, J. et collaborateurs. (1996). Notre diversité créatrice. Rapport de la Commission mondiale de la culture et du développement. Paris : Unesco.

Rahnema, M. (2004). Quand la misère chasse la pauvreté. Arles : Babel-Actes Sud.

Wicht, B. et Mondjanagni A. (2004). Diversité culturelle et mondialisation (p. 98-121). Paris : Autrement, Collection mutations no. 233.

\section{AUTEUR}

\section{THIERRY PARDO}

Actuellement étudiant à la maîtrise en éducation de l'Université du Québec à Montréal, où il traite des voies alternatives à l'éducation scolaire et passionné par les questions pédagogiques, il œuvre dans le domaine de l'éducation relative à l'environnement depuis une quinzaine d'années. Il est également l'auteur d'Héritages buissonniers consacré aux savoirs pédagogiques autochtones. 